$\underline{\underline{\text { IIIIIII }}}$ Open Access Articles

First Demonstration of a Self-Aligned GaN p-FET

The MIT Faculty has made this article openly available. Please share how this access benefits you. Your story matters.

\begin{tabular}{|l|l|}
\hline Citation & $\begin{array}{l}\text { Chowdhury, Nadim, Xie, Qingyun, Yuan, Mengyang, Rajput, Nitul S, } \\
\text { Xiang, Peng et al. 2019. "First Demonstration of a Self-Aligned GaN } \\
\text { p-FET." Technical Digest - International Electron Devices Meeting, } \\
\text { IEDM, 2019-December. }\end{array}$ \\
\hline As Published & $10.1109 /$ IEDM19573.2019.8993569 \\
\hline Publisher & Institute of Electrical and Electronics Engineers (IEEE) \\
\hline \hline Version & Author's final manuscript \\
\hline Citable link & https://hdl.handle.net/1721.1/137036 \\
\hline Terms of Use & Creative Commons Attribution-Noncommercial-Share Alike \\
\hline Detailed Terms & http://creativecommons.org/licenses/by-nc-sa/4.0/ \\
\hline
\end{tabular}




\title{
First Demonstration of a Self-Aligned GaN p-FET
}

\author{
Nadim Chowdhury ${ }^{\dagger 1}$, Qingyun Xie ${ }^{1}$, Mengyang Yuan ${ }^{1}$, Nitul S. Rajput ${ }^{2}$, Peng Xiang ${ }^{3}$, Kai Cheng ${ }^{3}$, \\ Han Wui Then ${ }^{4}$, and Tomás Palacios ${ }^{1}$ \\ ${ }^{1}$ Microsystems Technology Laboratories, Massachusetts Institute of Technology, Cambridge, MA 02139, U.S.A. ${ }^{2}$ Masdar Institute, \\ Khalifa University, Abu Dhabi 127788, U.A.E. ${ }^{3}$ Enkris Semiconductor, Inc., Suzhou, Jiangsu 215123, China. ${ }^{4}$ Intel Corporation, \\ Components Research, Technology Development Group, Hillsboro, OR 97124, U.S.A. `email: nadim@mit.edu
}

\begin{abstract}
In this work, we demonstrate a self-aligned p-FET with a $\mathrm{GaN} / \mathrm{Al}_{0.2} \mathrm{Ga}_{0.8} \mathrm{~N}(20 \mathrm{~nm}) / \mathrm{GaN}$ heterostructure grown by metal-organic-chemical vapor deposition (MOCVD) on $\mathrm{Si}$ substrate. Our $100 \mathrm{~nm}$ channel length device with recess depth of $70 \mathrm{~nm}$ exhibits a record ON-resistance of $400 \Omega \cdot \mathrm{mm}$ and ONcurrent over $5 \mathrm{~mA} / \mathrm{mm}$ with ON-OFF ratio of $6 \times 10^{5}$ when compared with other p-FET demonstrations based on $\mathrm{GaN} / \mathrm{AlGaN}$ heterostructure. The device shows E-mode operation with a threshold voltage of $-1 \mathrm{~V}$, making it a promising candidate for GaN-based complementary circuit that can be integrated on a Silicon platform. A monolithically integrated $\mathrm{n}$-channel transistor with $\mathrm{p}-\mathrm{GaN}$ gate is also demonstrated. The potential of the reported p-FET for complementary logic application is evaluated through industrystandard compact modeling and inverter circuit simulation.
\end{abstract}

\section{INTRODUCTION}

In order to keep up with the ever increasing demand of cloud computing, data centers and electric vehicles, power electronic circuits need to be more energy efficient and demonstrate higher power density levels. AlGaN/GaN High Electron Mobility Transistors (HEMTs) have shown great promise towards that end, thanks to their lower switching loss (attributed to lower terminal capacitances) and the higher switching speed enabled by their high electron mobility. However, in today's power electronic circuits, GaN power transistors are typically switched at relatively lower frequency (hundreds of $\mathrm{kHz}$ ) in order to avoid voltage instabilities caused by the parasitic inductance between the Silicon driver chip and that GaN switch [1]. To eliminate this parasitic inductance, it is necessary to monolithically integrate GaN-based complementary driver circuits with the GaN power transistors. However, this has been difficult until now due to the limited performance of p-channel GaN transistors. Most of the two-dimensional hole gas (2-DHG) based p-FETs demonstrated in the literature show normally-ON operation with ON-resistance over $1 \quad \mathrm{k} \Omega \cdot \mathrm{mm} \quad$ [2]-[6]. GaN/AlInGaN heterostructure-based p-FET shows low ONresistance because of higher 2-DHG density and hole mobility but with D-mode operation [7]. Ref. [8] demonstrated E-mode p-FET based on GaN/AIN heterostructure with $\mathrm{R}_{\mathrm{ON}}$ of 640 $\Omega \cdot \mathrm{mm}$. However, n-FET integration with this p-FET requires regrowth. While most of the demonstrations are on Sapphire substrate, Ref. [9] was the first demonstration on Silicon substrate. The utilization of GaN-on-Si platform offers lower cost, availability of 200 -mm-diameter substrates, and potential to integrate with high performance logic and analog functionalitv.
A key reason for the poor performance in p-FETs is their low hole mobility [2]. This could be circumvented by aggressive device scaling. A self-aligned device with scaled gate length and no access region is an effective way to achieve greater performance in terms of on resistance. In this work, we demonstrate a novel fabrication technique to fabricate a selfaligned gate-recessed p-FET with $\mathrm{Lg}_{\mathrm{g}}=100 \mathrm{~nm}$. The demonstrated technology provides a pathway for high performance GaN-based p-FET which can enable GaN-based complementary circuit technology on a $\mathrm{Si}$ substrate. A monolithically integrated $\mathrm{n}$-channel is also fabricated. The potential of the demonstrated p-FET for complementary logic application is evaluated through circuit simulation employing the MIT Virtual Source GaN-FET (MVSG) model [10].

\section{Epitaxial Structure}

The epitaxial stack used in this work was grown by Enkris Semiconductor, Inc. on a 6 inch, 1-mm-thick Si (111) substrate using MOCVD method. The structure is as follows, $20 \mathrm{~nm} \mathrm{p}^{++}$GaN (Mg: $6 \times 10^{19} \mathrm{~cm}^{-3}$ with $2-3 \%$ activation at room temperature), $50 \mathrm{~nm} \mathrm{p}-\mathrm{GaN}\left(\mathrm{Mg}: 10^{19} \mathrm{~cm}^{-3}\right), 20 \mathrm{~nm}$ UID-GaN (Si: $\left.5 \times 10^{16} \mathrm{~cm}^{-3}\right), 20 \mathrm{~nm} \mathrm{Al} l_{0.2} \mathrm{Ga}_{0.8} \mathrm{~N}\left(\mathrm{Si}: 5 \times 10^{16} \mathrm{~cm}^{-3}\right), 150 \mathrm{~nm}$ UID-GaN (Si: $5 \times 10^{16} \mathrm{~cm}^{-3}$ ), $3.8 \mu \mathrm{m}$ buffer and Si substrate (see Fig. 1 (a)). Fig. 1(b) shows the Transmission Electron Microscopy (TEM) image of the GaN/AlGaN/GaN double heterostructure. The p-channel FET uses the polarizationinduced 2-DHG at the interface of the top UID-GaN/AlGaN heterostructure. The top $\mathrm{p}^{++}-\mathrm{GaN}$ layer enables good ohmic contact. The $20 \mathrm{~nm}$ UID-GaN layer that separates the p-GaN and $\mathrm{AlGaN}$ layers helps to achieve higher hole mobility. Using Hall measurement, the 2-DHG density and hole drift mobility are measured to be $8 \times 10^{12} \mathrm{~cm}^{-2}$ and $11 \mathrm{~cm}^{2} / \mathrm{V} \cdot \mathrm{s}$ respectively. Fig. 1(c) shows the band diagram of the epitaxial structure exhibiting the Fermi level crossing the valence band energy level giving rise to 2-DHG (as shown in Fig. 1 (d)).

\section{Self-Aligned Transistor Fabrication}

Fig. 1(e) shows the key processing steps for fabricating the self-aligned device. First, electron beam lithography is used for the formation of source and drain contacts. Then, a $\mathrm{Ni}(20$ $\mathrm{nm}) / \mathrm{Au}(30 \mathrm{~nm}) / \mathrm{Ni}(20 \mathrm{~nm})$ metal stack are deposited using electron beam evaporation and lifted off in acetone. Next, a blank etch is performed by $\mathrm{Cl}_{2} / \mathrm{BCl}_{3}$-based Inductively Coupled Plasma-Reactive Ion Etching (ICP-RIE) process. This step helps to form the gate recess. Here, the top $20 \mathrm{~nm} \mathrm{Ni}$ on the source and drain metal serves as the etch mask and protects the ohmic contact from the etch damage. The etch depth is controlled by the etch time (see Fig. 1(f)). Then, the mesa etch is performed by 
$\mathrm{Cl}_{2} / \mathrm{BCl}_{3}$-based ICP-RIE process using photoresist mask. The photoresist is then removed by oxygen plasma etching. Following the etch steps, the sample is dipped into heated Tetramethylammonium hydroxide (TMAH) for about $15 \mathrm{~min}$ in order to remove the photoresist and reduce the etch-induced roughness. After that, $\sim 30 \mathrm{~nm}$ of $\mathrm{Al}_{2} \mathrm{O}_{3}$ gate dielectric is deposited by Atomic Layer Deposition (ALD). Finally, a Ni (20 $\mathrm{nm}) / \mathrm{Au}(100 \mathrm{~nm})$ gate metal electrode is formed by e-beam evaporation and lift-off technique. Fig. 1(g) exhibits the Scanning Transmission Electron Microscopy (STEM) image of the cross-section of the fabricated device. Fig. 1(h) shows the smooth interface between the $\mathrm{GaN}$ and gate dielectric attesting to the high quality of gate recess process with low surface roughness.

\section{RESULTS AND DISCUSSION}

Fig 2 (a) shows the transfer characteristics of the p-FET with $\mathrm{I}_{\mathrm{ON}} / \mathrm{I}_{\mathrm{OFF}}=6 \times 10^{5}$ and low gate leakage in the range of $1 \mathrm{nA} / \mathrm{mm}$. In the OFF-state, the drain leakage current is limited by gate leakage. The high gate leakage in the OFF-state is because of the high electric field that exists across the gate dielectric at the sidewall of the gate recess, and between the gate and drain terminals. Fig. 2(b) shows the $\mathrm{I}_{\mathrm{DS}} v s \mathrm{~V}_{\mathrm{GS}}$ characteristics in the linear scale, demonstrating E-mode operation with a threshold voltage of $-1 \mathrm{~V}$. Fig. 2(c) shows the $\mathrm{g}_{\mathrm{m}} v s \mathrm{~V}_{\mathrm{GS}}$ characteristics of the p-FET showing a maximum transconductance of $0.9 \mathrm{mS} / \mathrm{mm}$ at $\mathrm{V}_{\mathrm{DS}}=-1.5 \mathrm{~V}$ and $\mathrm{V}_{\mathrm{GS}}=-4 \mathrm{~V}$. Fig. 2(d) shows the output characteristics of the self-aligned p-FET with $\mathrm{R}_{\mathrm{ON}}$ of $\sim 400$ $\Omega \cdot \mathrm{mm}$ at $\mathrm{V}_{\mathrm{GS}}=-7 \mathrm{~V}$ (which is calculated by taking an inverse slope at low $\mathrm{V}_{\mathrm{DS}}$ regime of the output characteristics). The figure also shows an ON-current density of $>5 \mathrm{~mA} / \mathrm{mm}$ at $\mathrm{V}_{\mathrm{GS}}=-7 \mathrm{~V}$ and $\mathrm{V}_{\mathrm{DS}}=-5 \mathrm{~V}$.

Fig. 3 shows the output characteristics of the self-aligned pFET with $300 \mathrm{~nm}$ channel length for different recess depths. The depths are estimated based on time of etch using a calibrated etch recipe (as shown in Fig 1(f)). The device performance is found to be dependent on recess depth - shallower recess depth results in more ON-current but weaker current modulation with respect to gate voltage.

Fig. 4(a)-(b) shows the IDS $v s V_{D S}$ characteristics of selfaligned p-FET with $500 \mathrm{~nm}$ channel length, at room and cryogenic temperatures, respectively. A $\sim 20 \%$ drop in ONcurrent at low temperature could be observed from the results, which is attributed to the increase in contact resistance at low temperature because of low $\mathrm{Mg}$ activation. This is attested in Fig. 4(c) which shows the linear TLM measurements both at room and cryogenic temperatures.

A key advantage of the proposed p-FET device architecture is that it allows for the monolithic integration of self-aligned $\mathrm{p}$ FET and n-channel GaN FET on the same Si wafer (Fig. 5(a)). The $\mathrm{I}_{\mathrm{DS}} v s \mathrm{~V}_{\mathrm{GS}}$ characteristics of the fabricated n-channel FET are shown in Fig. 5(b). Fig. 5(c) shows the output characteristics of the n-channel transistor with an RON of $19 \Omega \cdot \mathrm{mm}$ and ONcurrent over $200 \mathrm{~mA} / \mathrm{mm}$. A threshold voltage of $0.2 \mathrm{~V}$ is obtained from the monolithically integrated n-FET. A p-GaN gated device without the $20 \mathrm{~nm}$ UID GaN layer and thinner AlGaN layer $(\sim 15 \mathrm{~nm})$, which is typically used for E-mode p-
GaN gated $\mathrm{AlGaN} / \mathrm{GaN}$ HEMTs, yields a threshold voltage of 2 $\mathrm{V}$ (Fig. 5(d)). A more positive threshold voltage for $\mathrm{p}-\mathrm{GaN}$ gated n-FET using the epitaxial layer of Fig. 1(a), could be achieved by FinFET or nanoribbon structure [11].

Fig. 6 benchmarks the performance of the fabricated selfaligned p-FET in this work with other p-channel transistors reported in the literature in terms of ON-resistance and ON-OFF ratio. As shown, the device in this work shows record performance in terms of ON-resistance and ON-OFF ratio when compared with $\mathrm{GaN} / \mathrm{AlGaN}$ and $\mathrm{InGaN} / \mathrm{GaN}$ based p-FETs. It should be noted that, GaN/AlInGaN based p-FETs show slightly lower resistance compared to the self-aligned p-FETs in this work because of very high 2-DHG density provided by large negative polarization charge at the III-N heterointerface but with normally-ON operation. The p-FET reported in this work has the additional advantage of having a 2-DEG beneath the 2-DHG which facilitates on-chip n-channel transistor with the same epistructure without any regrowth. The reported p-FET also shows E-mode operation, which is absent in most of the devices reported in the literature so far.

To evaluate the potential of the reported p-FET and n-FET monolithic integration, for GaN-based complementary logic applications, circuit simulation was conducted using Cadence Virtuoso and compact models for the devices obtained using the industry-standard MVSG compact model. The inverter circuit and simulation methodology are presented in Fig. 7(a). A DC bias of $5 \mathrm{~V}$ was used. The n-FET and p-FET have gate widths of $25 \mu \mathrm{m}$ and $250 \mu \mathrm{m}$, respectively. The simulated voltage transfer curves for the complementary logic inverter are presented in Fig. $7(\mathrm{~b})$, which shows a maximum voltage gain of 48 at $\mathrm{V}_{\mathrm{IN}}=0.68$ $\mathrm{V}$. The $\mathrm{DC}$ current drawn by the inverter $\left(\mathrm{I}_{\mathrm{DD}}\right)$ has a peak of 0.52 $\mathrm{mA}$ at $\mathrm{V}_{\mathrm{IN}}=0.70 \mathrm{~V}$, which is the switching point of the inverter. The switching behavior of the inverter was studied by applying a capacitive load of $35 \mathrm{pF}$. As shown in Fig. 7(c)-(d), the fall and rise times of the circuit are $60 \mathrm{~ns}$ and $301 \mathrm{~ns}$, respectively. The longer rise time is mainly due to the high $\mathrm{R}_{\mathrm{ON}}$ of the p-FET.

\section{Conclusion}

In this work, we demonstrate self-aligned GaN p-FET with $100 \mathrm{~nm}$ gate length for the first time, with ON-OFF ratio $6 \times 10^{5}$ and threshold voltage of $-1 \mathrm{~V}$. The demonstrated device shows record ON-resistance of $400 \Omega \cdot \mathrm{mm}$ with ON-current of $>5$ $\mathrm{mA} / \mathrm{mm}$ when compared with other GaN/AlGaN heterostructure-based p-FET demonstrations. The capability for monolithic integration of this $\mathrm{p}$-FET with $\mathrm{p}-\mathrm{GaN}$ gated n-FET on a Si substrate makes this technology an ideal candidate for GaN complementary logic for integrated power driver circuits.

\section{REFERENCES}

[1] F. Luo et al., Proc. IEEE APEC, pp. 537-544, Mar. 2014.

[2] R. Chu et al., IEEE EDL, vol. 37, no. 3, pp. 269-271, Mar. 2016.

[3] T. Zimmermann et al., IEEE EDL, vol. 25, pp. 450-452, Jul. 2004.

[4] K. Zhang et al., Sci. Rep., vol. 6, pp. 23683, Mar. 2016.

[5] A. Nakajima et al., Proc. ISPSD, pp. 241-244, Jun. 2014.

[6] M. Shatalov et al., IEEE EDL, vol. 23, no. 8, pp. 452-454, Aug. 2002.

[7] B. Reuters et al., J. Phys. D., vol. 47, no. 17, pp. 175103, Apr. 2014.

[8] S. J. Bader et al., IEEE EDL, vol. 39, no. 12, pp. 1848-1851, Dec. 2018.

[9] N. Chowdhury et al., IEEE EDL, vol. 40, no. 7, pp. 1036-1039, Jul. 2019.

[10] U. Radhakrishna et al., Proc. IEDM, pp. 13.6, Dec. 2012.

[11] B. Lu et al., IEEE EDL, vol. 33, no. 3, pp. 360-362, Jan. 2012. 
(a)

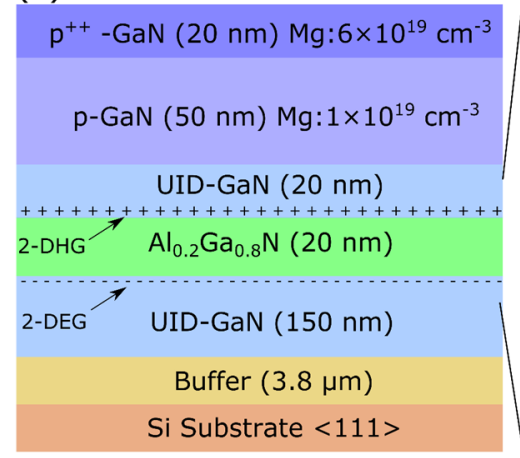

(b)
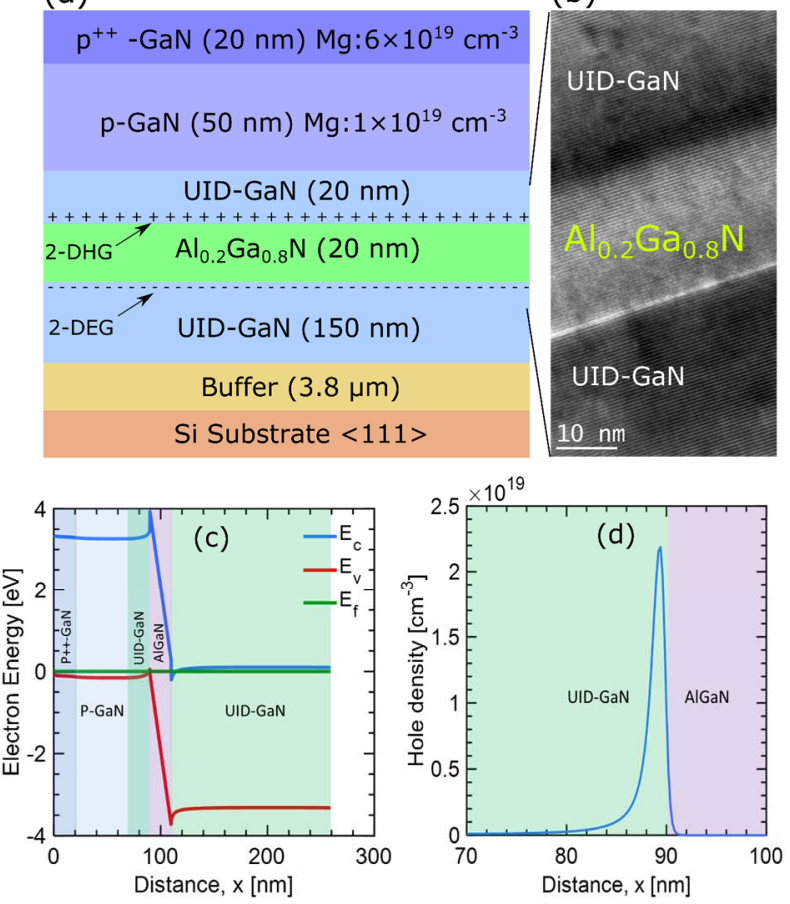

$\mathrm{Cl}_{2}+\mathrm{BCl}_{3}$ Plasma
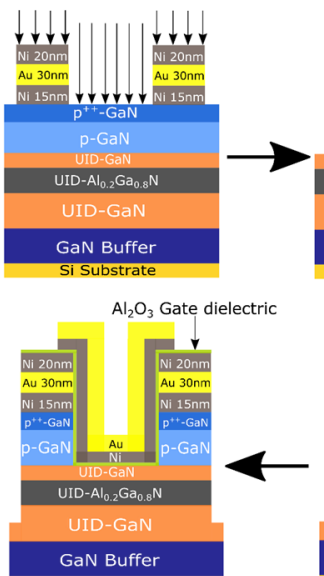

GaN Buffer

Si Substrate

2. Electron beam lithography

3. E-beam evaporation of $\mathrm{Ni} / \mathrm{Au} / \mathrm{Ni}$ and

lift-off (Source/Drain formation)

4. Gate recess etch with $\mathrm{Cl} 2$ and $\mathrm{BCl} 3$ plasma (Global etch)

5. Photo lithography and Mesa etch

6. Heated TMAH dip for 5 mins

7. ALD of gate dielectric

8. E-beam lithography (Gate)

9. E-beam evaporation of $\mathrm{Ni} / \mathrm{Au}$ and lift-off. (Gate formation) (f)

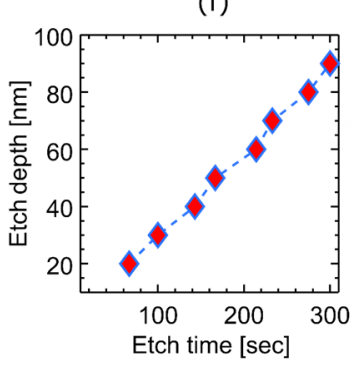

(g)

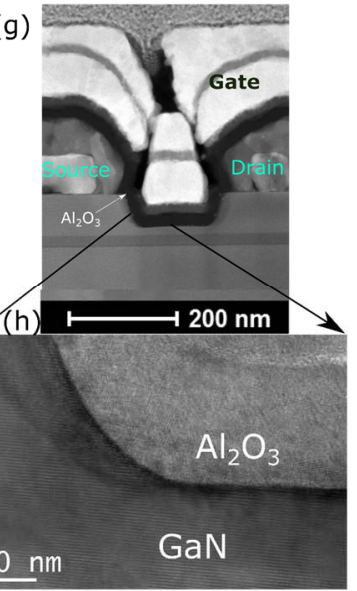

Fig. 1: (a) MOCVD grown epitaxial structure on 6-inch Si wafer. (b) TEM image of the GaN/AlGaN/GaN double heterostructure. (c) Simulated electron energy

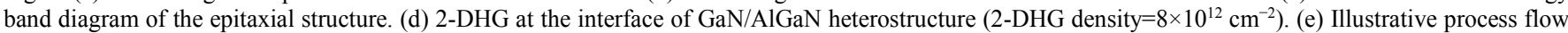
for the formation of self-aligned E-mode p-channel FET. Here, the gate recess is enabled by $20 \mathrm{~nm}$ of Ni on top of Ni/Au ohmic contact which serves as the etch mask and protects the ohmic contact from the plasma damage. (f) Etch depth $v s$ etch time for the gate recess etch. (g) STEM cross-section view of the fabricated self-aligned device. (h) TEM image of the GaN / gate dielectric interface showing the high quality of gate recess with low surface roughness.
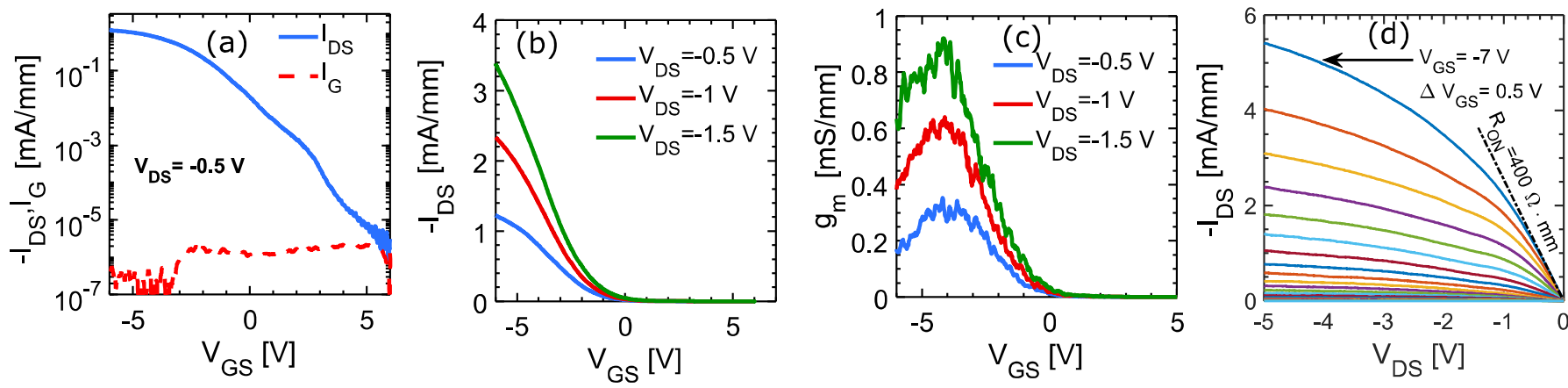

Fig. 2: (a) Semi-log plot of $I_{D S} v S V_{G S}$ at $V_{D S}=-0.5 \mathrm{~V}$ demonstrating an ON-OFF ratio of $6 \times 10^{5}$. In the ON-state, the gate leakage current is quite small in the range of $1 \mathrm{nA} / \mathrm{mm}$. However, an increase in gate leakage is observed in the OFF-state of the device, mainly because of the high electric field at the drain-end of the transistor. (b) Linear scale plot of $\mathrm{I}_{\mathrm{DS}} v s \mathrm{~V}_{\mathrm{GS}}$ demonstrating a threshold voltage of $-1 \mathrm{~V}$. (c) Measured $\mathrm{g}_{\mathrm{m}} v s \mathrm{~V}_{\mathrm{GS}}$ characteristics with a peak transconductance of $0.9 \mathrm{mS} / \mathrm{mm}$. (d) Measured I $\mathrm{DS}_{\mathrm{DS}} \mathrm{V}_{\mathrm{DS}}$ characteristics demonstrating record ON-resistance of $400 \Omega \cdot \mathrm{mm}$.

Increase in gate recess depth
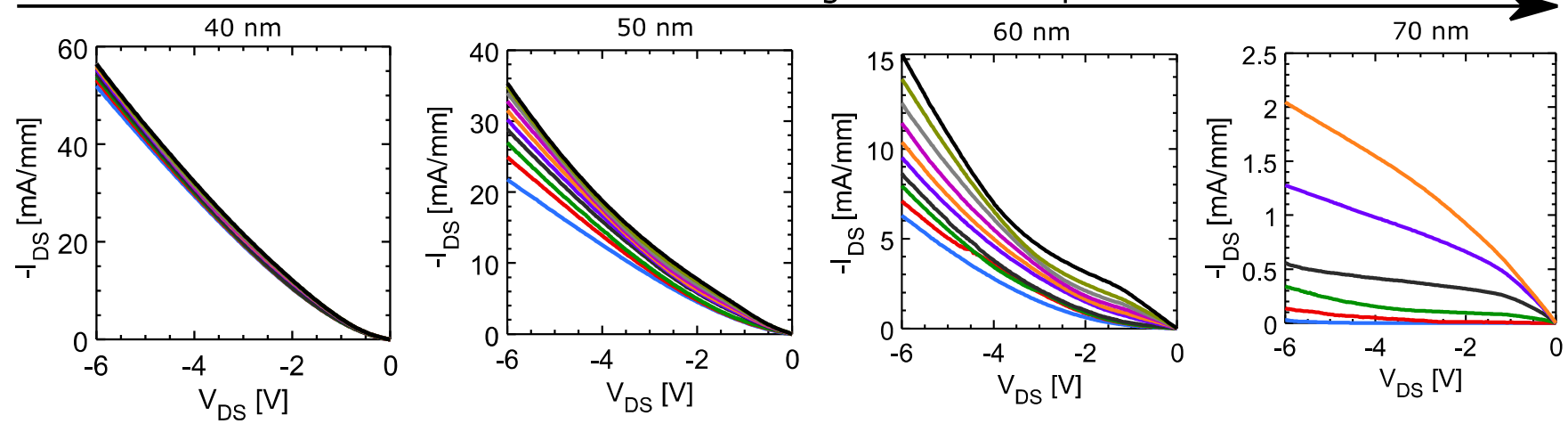

Fig. 3: $I_{D S} v s V_{D S}$ characteristics of the self-aligned p-FET with different recess depths, demonstrating that a shallow recess yields higher ON-current at the expense of low ON-OFF ratio (here, $\mathrm{V}_{\mathrm{GS}, \min }=-5 \mathrm{~V}$ and $\Delta \mathrm{V}_{\mathrm{GS}}=1 \mathrm{~V}$ ). Devices with less than $70 \mathrm{~nm}$ recess depth could not be fully turned off because of the conduction through the un-etched $\mathrm{p}-\mathrm{GaN}$ beneath the gate. 

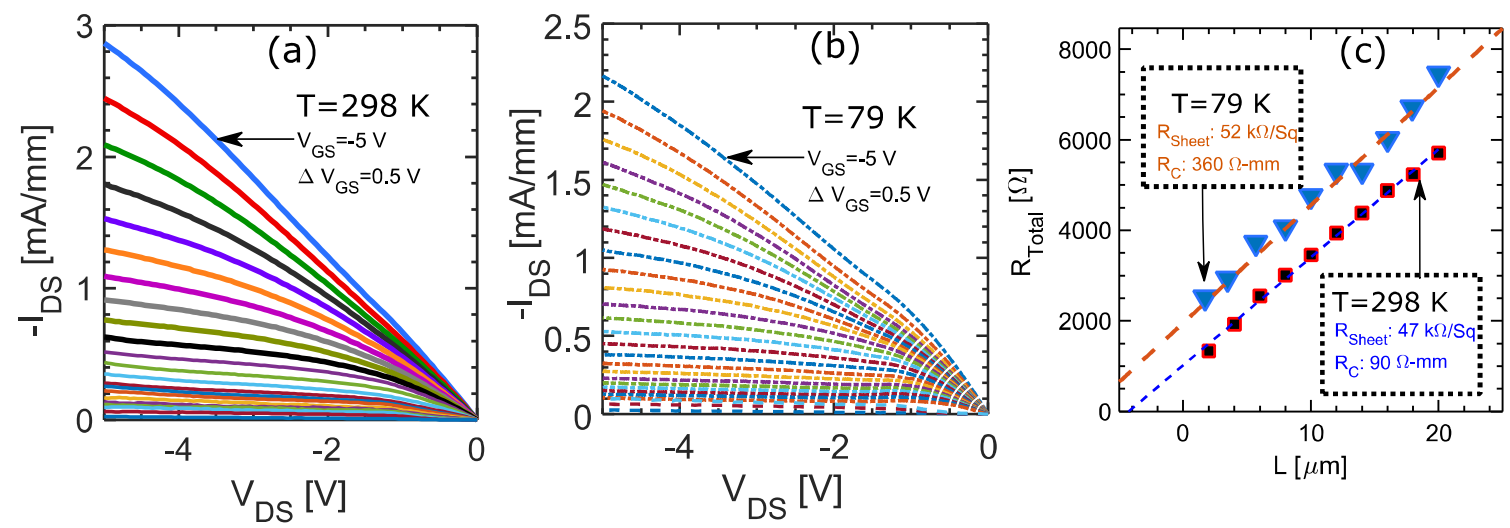

Fig. 4: $\mathrm{I}_{\mathrm{DS}} v s \mathrm{~V}_{\mathrm{DS}}$ characteristics of the self-aligned p-FET with $500 \mathrm{~nm}$ channel length and $70 \mathrm{~nm}$ recess depth at (a) room temperature $\mathrm{T}=298 \mathrm{~K}$ and at (b) cryogenic temperature $\mathrm{T}=79 \mathrm{~K}$. It is quite evident that at low temperature, drain current is reduced by $\sim 20 \%$ for the same gate voltage. This reduction in current can be explained in terms of the increase in contact resistance at low temperature. (c) Linear TLM measurement results at T=298 K and T=79 K. As shown here, a reduction in temperature leads to an increment of both the sheet resistance and the contact resistance. This is because of the lower activation ratio of $\mathrm{Mg}$ at low temperature.
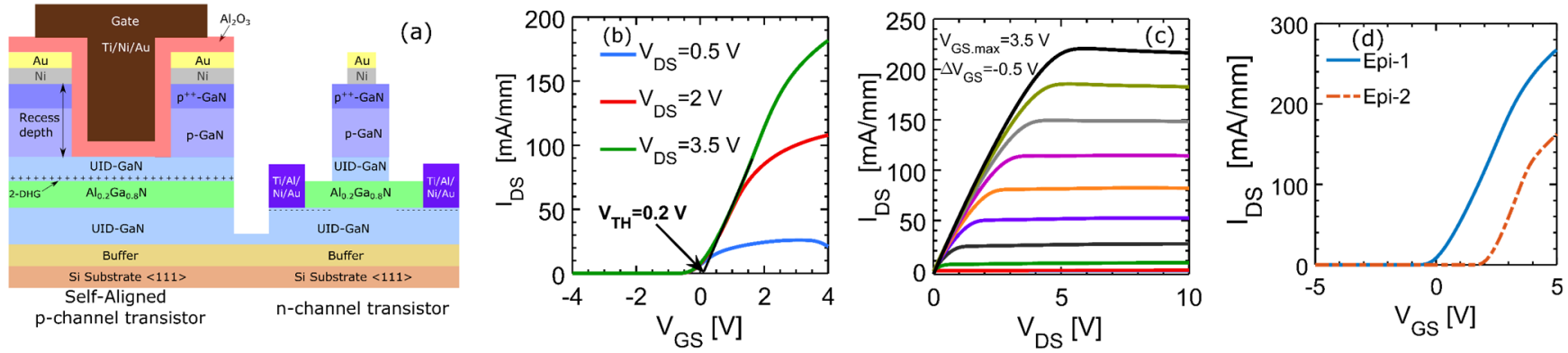

Fig. 5: (a) Monolithic integration of self-aligned p-FET and p-GaN gated n-FET. (b) $I_{D S} v s V_{G S}$ characteristics of the $n$-FET in linear scale demonstrating a threshold voltage of $0.2 \mathrm{~V}$. (c) I $\mathrm{I}_{\mathrm{DS}} v s \mathrm{~V}_{\mathrm{DS}}$ characteristics of the n-channel FET. (d) $\mathrm{I}_{\mathrm{DS}} v s \mathrm{~V}_{\mathrm{GS}}$ characteristics of the p-GaN gated n-FETs fabricated using two different epitaxial structures described as follows, Epi-1: same as epi-structure used in this work (see Fig. 1(a)), Epi-2: Epi-structure used for conventional Emode $\mathrm{p}$-GaN gated AlGaN/GaN HEMT - $70 \mathrm{~nm}$ p-GaN $\left(\mathrm{Mg}: 10^{19} \mathrm{~cm}^{-3}\right), 15 \mathrm{~nm} \mathrm{Al} \mathrm{l}_{0.2} \mathrm{Ga}_{0.8} \mathrm{~N}\left(\mathrm{Si}: 5 \times 10^{16} \mathrm{~cm}^{-3}\right), 150 \mathrm{~nm} \mathrm{UID-GaN}\left(\mathrm{Si}: 5 \times 10^{16} \mathrm{~cm}{ }^{-3}\right)$. This reflects that insertion of UID-GaN layer and thickening of AlGaN layer shifts the threshold voltage towards zero which can be resolved by using FinFET structure.

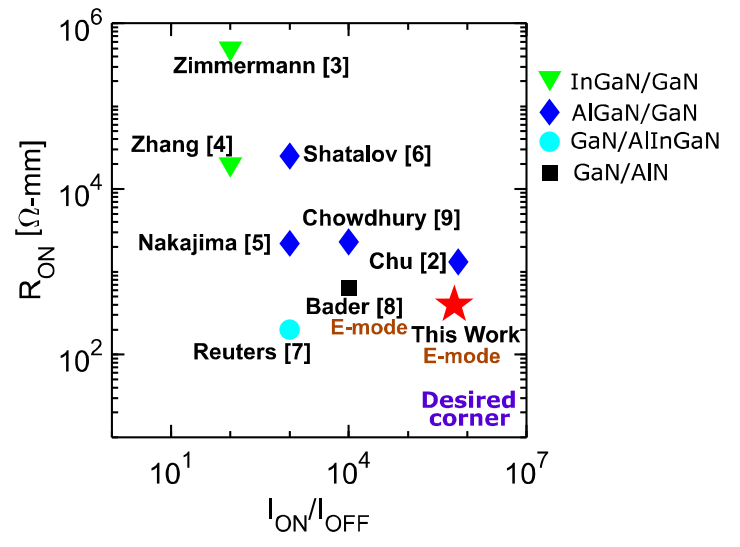

Fig. 6: Benchmarking of the reported self-aligned p-FET with other demonstrated p-FETs in the literature in terms of ON-resistance and ONOFF ratio. It is quite evident that the reported device is closest to the desired corner exhibiting an ON-resistance of $400 \Omega \cdot \mathrm{mm}$ with excellent ON-OFF ratio of $6 \times 10^{5}$. The p-FET in this work enables easy integration with on-chip n-FET without any regrowth, as illustrated in Fig. 5(a). The device is E-mode with $-1 \mathrm{~V}$ threshold voltage. This work shows record $\mathrm{ON}$-resistance and ON-OFF ratio for any GaN-based E-mode p-FET, making it a promising candidate for GaN-based complementary logic.

(a)
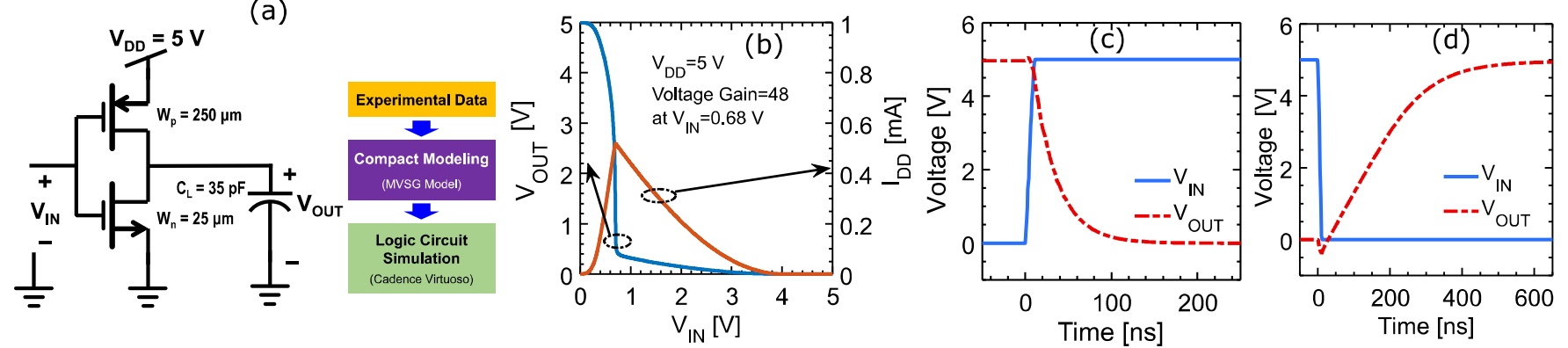

Fig. 7: (a) Circuit diagram and methodology used in this work to simulate the GaN-based complementary logic inverter. (b) Voltage transfer curve of the GaNbased complementary logic inverter. The n-FET and p-FET have gate widths of $25 \mu \mathrm{m}$ and $250 \mu \mathrm{m}$, respectively. Simulated waveforms showing the (c) fall and (d) rise edge of the output signal from the inverter. The rise/fall time of the input signal is $10 \mathrm{~ns}$. The fall and rise times of the output signal are $60 \mathrm{~ns}$ and 301 ns, respectively. 